\section{Layered steered space-time codes and their capacity}

\section{El-Hajjar and L. Hanzo}

A capacity analysis of a multifunctional multiple-input multiple-output system that combines the benefits of vertical Bell Labs space-time scheme, space-time block codes and beamforming is presented.

Introduction: Recent information theoretic studies [1] have revealed that employing a multiple-input multiple-output (MIMO) scheme significantly increases the capacity of the system. In [2], Wolniansky et al. proposed the popular multilayer MIMO structure, known as the vertical Bell Labs layered space-time (V-BLAST) scheme that is capable of providing a tremendous increase of a specific user's effective bit-rate, since it was designed for achieving a MIMO-aided multiplexing gain. On the other hand, space-time codes (STC) [3] were designed for a high diversity gain. Furthermore, it was proposed in [4] to combine the benefits of these two techniques for the sake of providing both diversity as well as multiplexing gains. Moreover, beamforming [5] constitutes an effective technique for increasing the antenna gain in the desired user's direction and thus minimising the effects of interference in the other users' directions. In this Letter, we propose a new generalised MIMO, which we refer to as layered steered space-time code (LSSTC) that combines the benefits of VBLAST, STC and beamforming for improving the achievable system performance and deriving the capacity limits for this powerful new scheme.

Layered steered space-time codes: A high-level block diagram of the proposed scheme is shown in Fig. 1. The antenna architecture employed in Fig. 1 has $M$ transmit antenna arrays (AA) spaced sufficiently far apart in order to experience independent fading and hence achieve transmit diversity. A number of elements $L$ of each of the AAs are spaced at a distance of $d=\lambda / 2$ for the sake of achieving beamforming. Furthermore, the receiver is equipped with $N \geq M$ antennas. According to Fig. 1, a block of $B$ input information bits is serial-to-parallel converted to $K$ groups of bit streams of length $B_{1}$, $B_{2}, \ldots, B_{K}$, where $B_{1}+B_{2}+\ldots+B_{K}=B$. Each group of $B_{k}$ bits, $k \in[1, K]$, is then encoded by a component space-time code $\mathrm{STC}_{k}$ associated with $m_{k}$ transmit AAs, where $m_{1}+m_{2}+\ldots+m_{K}=M$. We consider transmissions over a correlated narrowband Rayleigh fading channel, associated with a normalised Doppler frequency of $f_{D}=f_{d} T_{s}=0.01$, where $f_{d}$ is the Doppler frequency and $T_{s}$ is the symbol duration. The complex additive white Gaussian noise (AWGN) has a zero mean and a variance of $N_{0} / 2$ per dimension.

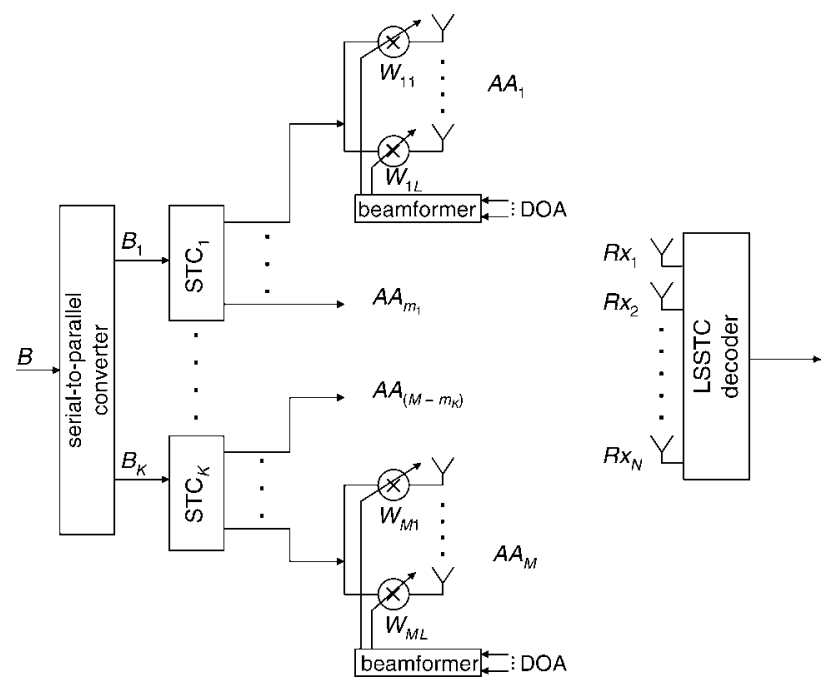

Fig. 1 Proposed system model

The $L$-dimensional spatio-temporal (ST) channel impulse response (CIR) vector spanning the $m$ th transmitter AA, $m \in[1, \ldots, M]$, and the $n$th receiver antenna, $n \in[1, \ldots, N]$, can be expressed as $\boldsymbol{h}_{n m}(t)=$ ${ }_{n m}(t)=\boldsymbol{a}_{n m}(t) \delta\left(t-\tau_{k}\right)=\left[a_{n m, 0}(t), \ldots, a_{n m,(L-1)}(t) \tau_{k}\right)$, where $\tau_{k}$ is the signal's delay, $a_{n m, l}(t)$ is the CIR with respect to the $m n$th link and the $l$ th element of the $m$ th AA. Based on the assumption that the array elements are separated by half a wavelength, we have $\boldsymbol{a}_{n m}(t)=$ $\alpha_{n m}(t) \cdot \boldsymbol{d}_{n m}$, where $\boldsymbol{a}_{n m}(t)$ is a Rayleigh faded envelope, $\boldsymbol{d}_{n m}=[1$, $\left.\exp \left(j\left[\pi \sin \left(\Psi_{n m}\right)\right]\right), \ldots, \exp \left(j\left[(L-1) \pi \sin \left(\Psi_{n m}\right)\right]\right)\right]^{T}$ and $\Psi_{n m}$ is the $n m$ th link's direction of arrival (DOA).

The received baseband data matrix $\boldsymbol{Y}$ can be expressed as $\boldsymbol{Y}=\boldsymbol{H} \boldsymbol{W} \boldsymbol{X}$ $+\boldsymbol{N}$, where $\boldsymbol{N}$ denotes the AWGN matrix and $\boldsymbol{H}$ is an $(N \times M)$ matrix whose entries are $\boldsymbol{h}_{n m}$. Furthermore, $\boldsymbol{W}$ is a diagonal weight matrix, whose diagonal entry $\boldsymbol{w}_{m n}$ is the $L$-dimensional weight vector for the $m$ th beamformer AA and the $n$th receive antenna. Let $\boldsymbol{w}_{m n}=\boldsymbol{d}_{n m}^{\dagger}$; then the received signal can be expressed as $\boldsymbol{Y}=L \tilde{\boldsymbol{H}} \boldsymbol{X}+\boldsymbol{N}$, where $\tilde{\boldsymbol{H}}$ is an $(N \times M)$ matrix whose entries are $\alpha_{n m}$. Moreover, $\boldsymbol{Y}$ can be written as $\boldsymbol{Y}=L \sum_{k=1}^{K} \tilde{\boldsymbol{H}}_{k} \boldsymbol{x}_{k}+\boldsymbol{N}$, where $\boldsymbol{x}_{k}$ represents the component STC used at layer $k$, with $k \in[1, \ldots, K]$. The most beneficial decoding order of the STC layers is determined on the basis of detecting the higher-power layer first for the sake of a higher correct detection probability. For simplicity let us consider the case of $K=2$ STBC layers and that layer 1 is detected first, which allows us to eliminate the interference caused by the signal of layer 2. However, the proposed concept is applicable to arbitrary STCs. For this reason, the decoder of layer 1 has to compute a matrix $\boldsymbol{Q}$, so that we have $\boldsymbol{Q} \cdot \tilde{\boldsymbol{H}}_{2}=0$. Therefore, the decoder computes an orthonormal basis for the left null space of $\tilde{\boldsymbol{H}}_{2}$ and assigns the vectors of the basis to the rows of $\boldsymbol{Q}$. Multiplying $\boldsymbol{Q}$ by $\boldsymbol{Y}$ suppresses the interference of layer 2 originally imposed on layer 1 and generates a signal, which can be decoded using maximum likelihood STBC detection. Then, the decoder subtracts the remodulated contribution of the decoded symbols of layer 1 from the composite twin-layer received signal. Finally, the decoder applies direct STBC decoding to the second layer, since the interference imposed by the first layer has been eliminated. This group-interference cancellation procedure can be generalised to any $M$ and $K$ values.

Capacity analysis: Upon using the decoding order of $(1,2, \ldots, K)$, group $k$ will have a diversity order of $m_{k} \times\left(N-M+m_{1}+m_{2}+\ldots\right.$ $\left.+m_{k}\right)=m_{k} \times N_{k}$. Thus, the LSSTC-decoded signal of layer $k$ can be described as $y_{k}=L \sum_{\tau=1}^{N_{k}} \quad \sum_{t=1}^{m_{k}} \quad \alpha_{r t} x_{k}+\Delta_{k}=\sum_{r=1}^{N_{k}} \quad \chi_{2 m_{k} r}^{2} x_{k}+\Delta_{k}$, where $\chi_{2 m_{k} r}^{2}=L \sum_{t=1}^{m_{k}} \alpha_{r t}$ represents a chi-squared distributed random variable having $2 m_{k}$ degrees of freedom and $\Delta_{k}$ is the AWGN after decoding having a noise variance of $\chi_{2 m_{l} r}^{2} N_{0} / 2$ per dimension. Let $y=\left(y_{1}, y_{2}, \ldots, y_{K}\right), x=\left(x_{1}, x_{2}, \ldots, x_{K}\right)$ and note that with $K$ groups there are $D=F^{K}$ number of possible F-ary phasor combinations where $F$-ary signalling is used for transmission. Thus, the achievable capacity of the proposed MIMO system for transmission over the discrete-input continuous-output memoryless channel (DCMC) can be derived from that of the discrete memoryless channel as $[6,7]$ :

$$
\begin{aligned}
C_{D C M C}= & \max _{p\left(\mathrm{x}_{1}\right), \cdots, p\left(\mathrm{x}_{D}\right)} \sum_{d=1}^{D} \int_{-\infty}^{+\infty} p\left(y / x_{d}\right) \cdot p\left(x_{d}\right) \\
& \times \log _{2}\left(\frac{p\left(y / x_{d}\right)}{\sum_{v=1}^{D} p\left(y / x_{v}\right) \cdot p\left(x_{v}\right)}\right) \cdot d y[\mathrm{bit} / \mathrm{sym}]
\end{aligned}
$$

where $x_{d}$ represents the $d$ th phasor out of the $D$ possible phasor combinations, and

$$
\begin{aligned}
p(y / x)= & \prod_{k=1}^{K} p\left(y_{k} / x_{k}\right) \text { and } p\left(y_{k} / x_{k}\right) \\
= & \frac{1}{\pi N_{0} \sum_{r=1}^{N_{k}} \chi_{2 m_{k} r}^{2}} \exp \left(\sum_{r=1}^{N_{k}} \frac{-\left(y_{k}-\chi_{2 m_{k} r}^{2} x_{k}\right)}{\chi_{2 m_{k} r}^{2} N_{0}}\right)
\end{aligned}
$$

Furthermore, $C_{D C M C}$ in (1) is maximised, when the transmitted symbols are equiprobably distributed, i.e. $p\left(x_{d}\right)=1 / D$. Finally, (1) can be simplified to

$$
\begin{aligned}
C_{D C M C} & =\log _{2}(D)-\frac{1}{D} \sum_{d=1}^{D} E \\
& \times\left[\log _{2}\left(\sum_{v=1}^{D} \exp \left(\Psi_{d v}\right)\right) \mid x_{d}\right][\mathrm{bit} / \mathrm{sym}]
\end{aligned}
$$


where $E[A \mid B]$ is the expectation of $A$ conditioned on $B$ and

$$
\Psi_{d v}=\sum_{k=1}^{K} \sum_{r=1}^{N_{k}}-\frac{\left|\chi_{2 m_{k} r}^{2}\left(x_{d k}-x_{v k}\right)+\Delta_{k}\right|^{2}+\left|\Delta_{k}\right|^{2}}{\chi_{2 m_{k} r}^{2} N_{0}}
$$

where $x_{d k}$ and $x_{v k}$ represent the $k$ th element in the vectors $x_{d}$ and $x_{v}$ respectively. Furthermore, the continuous-input continuous-output memoryless channel (CCMC) capacity of the proposed LSSTC scheme can be expressed as $[6,7]$ :

$$
C_{C C M C}=\sum_{k=1}^{K} E\left[\log _{2}\left(1+\sum_{r=1}^{N_{k}} \chi_{2 m_{k} r}^{2} \frac{S N R}{m_{k}}\right)\right][\mathrm{bit} / \mathrm{sym}]
$$

where we have $S N R=K \cdot R_{S T C} \cdot \log _{2}(F) \cdot E_{b} / N_{0}$ and $R_{S T C}$ is the rate of the specific STC used.

Results: We consider a system employing $M \times N=4 \times 4$ antennas and $K=2$ layers, in order to demonstrate the performance improvements achieved by a downlink (DL) scheme where a BS employing $M=4$ transmit antennas is communicating with a laptop receiver employing $N=4$ back plane antennas. The system employs QPSK modulation and considers transmission over a correlated Rayleigh fading channel. Fig. 2 shows the effect of increasing the DL BS beamforming gain by increasing the number of beam-steering elements $L$ in the AA, while maintaining the same number of AAs. As shown in the Figure, when the number of beam-steering elements $L$ increases, the achievable BER performance substantially improves. Fig. 3 quantifies the channel capacity limits of the proposed LSSTC scheme employing QPSK, $M=N=4$ and a variable value of $L$.

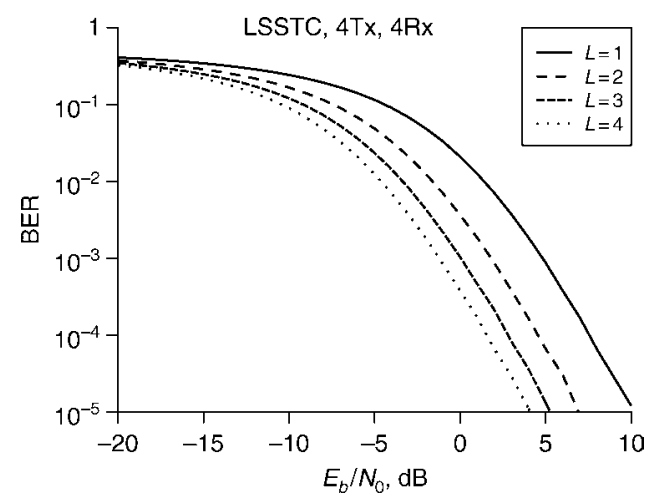

Fig. 2 BER performance of QPSK modulated $M \times N=4 \times 4$ LSSTC system for variable $L$

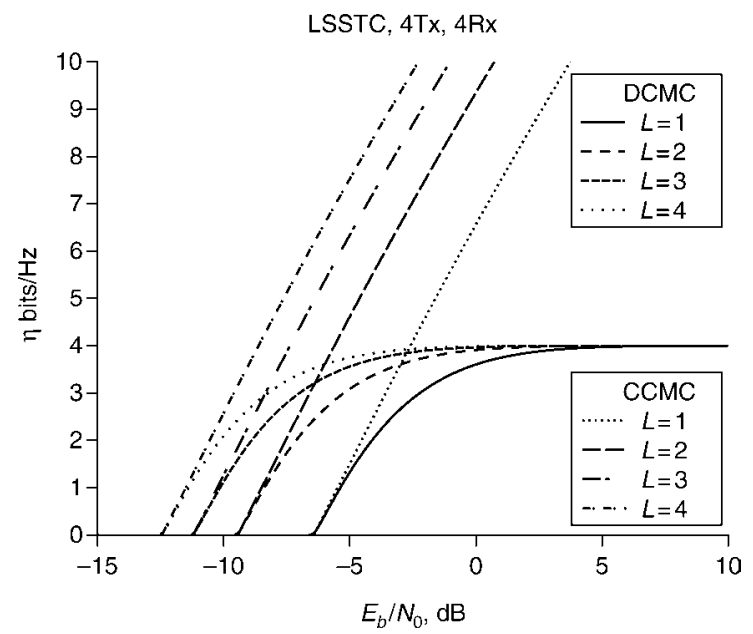

Fig. 3 Capacity of QPSK modulated $M \times N=4 \times 4$ LSSTC system for variable $L$
Acknowledgments: The financial support of Vodafone under the auspices of the Dorothy Hodgkin Postgraduate Award, as well as that of the EPSRC, UK, and of the European Union under the auspices of the Pheonix and Newcom projects is gratefully acknowledged.

(C) The Institution of Engineering and Technology 2007

26 March 2007

Electronics Letters online no: 20070862

doi: 10.1049/el:20070862

M. El-Hajjar and L. Hanzo (School of Electronics and Computer Science, University of Southampton, Southampton SO17 1BJ, United Kingdom)

E-mail: meh05r@ecs.soton.ac.uk

\section{References}

1 Foschini, G., and Gans, M.: 'On limits of wireless communications in a fading environment when using multiple antennas', Wirel. Pers. Commun., 1998, 5, pp. 311-335

2 Wolniansky, P., Foschini, G., Golden, G., and Valenzuela, R.: 'V-BLAST: an architecture for realizing very high data rates over the rich-scattering wireless channel'. Int. Symp. on Signals, Systems, and Electronics, September 1998, Pisa, Italy, pp. 295-300

3 Tarokh, V., Jafarkhani, H., and Calderbank, A.: 'Space-time block codes from orthogonal designs', IEEE Trans. Inf. Theory, 1999, 45, (5), pp. 1456-1467

4 Tarokh, V., Naguib, A., Seshadri, N., and Calderbank, A.: 'Combined array processing and space-time coding', IEEE Trans. Inf. Theory, 1999, 45, (4), pp. 1121-1128

5 Blogh, J., and Hanzo, L.: 'Third-generation systems and intelligent wireless networking: smart antennas and adaptive modulation' (John Wiley \& Sons - IEEE Press, 2002)

6 Gallager, R.: 'Information theory and reliable communication' (Wiley, New York, USA, 1968)

$7 \mathrm{Ng}$, S.X., and Hanzo, L.: 'On the MIMO channel capacity of multidimensional signal sets', IEEE Trans. Veh. Technol., 2006, 55, (2), pp. 528-536 